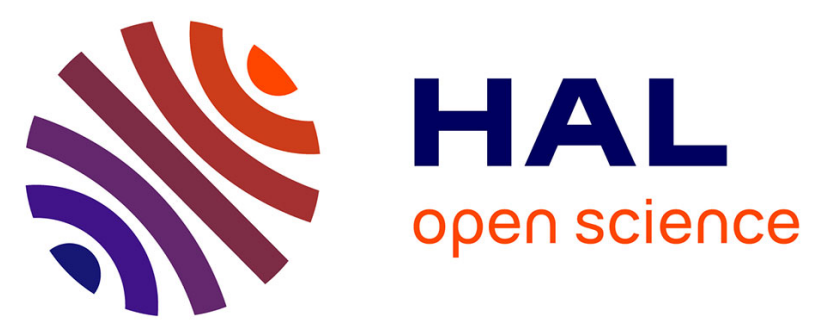

\title{
Density control of electrodeposited Ni nanoparticles/nanowires inside porous anodic alumina templates by an exponential anodization voltage decrease
}

Berndt Marquardt, Laurent Eude, Manoraham Gowtham, G. Cho, Hee Jeen

Jeong, Marc Châtelet, Costel Sorin Cojocaru, B. S. Kim, Didier Pribat

\section{To cite this version:}

Berndt Marquardt, Laurent Eude, Manoraham Gowtham, G. Cho, Hee Jeen Jeong, et al.. Density control of electrodeposited $\mathrm{Ni}$ nanoparticles/nanowires inside porous anodic alumina templates by an exponential anodization voltage decrease. Nanotechnology, 2008, 19, pp.405607. 10.1088/09574484/19/40/405607. hal-00795368

\section{HAL Id: hal-00795368 \\ https://hal.science/hal-00795368}

Submitted on 2 Mar 2013

HAL is a multi-disciplinary open access archive for the deposit and dissemination of scientific research documents, whether they are published or not. The documents may come from teaching and research institutions in France or abroad, or from public or private research centers.
L'archive ouverte pluridisciplinaire HAL, est destinée au dépôt et à la diffusion de documents scientifiques de niveau recherche, publiés ou non, émanant des établissements d'enseignement et de recherche français ou étrangers, des laboratoires publics ou privés. 


\title{
Density control of electrodeposited Ni nanoparticles/nanowires inside porous anodic alumina templates by an exponential anodization voltage decrease
}

\author{
B Marquardt ${ }^{1}$, L Eude ${ }^{1}$, M Gowtham ${ }^{1}$, G Cho ${ }^{1}$, H J Jeong ${ }^{1}$, M Châtelet ${ }^{1}$, C S Cojocaru ${ }^{1}$, B S Kim ${ }^{2}$ \\ and D Pribat ${ }^{1}$
}

${ }^{1}$ Laboratoire de Physique des Interfaces et des Couches Minces, Ecole Polytechnique, 91128,

Palaiseau, France

${ }^{2}$ Samsung Electronics, Giheung-Gu, Yongin-City, Gyeonggi-Do, 446-711, Korea

\begin{abstract}
Porous alumina templates have been fabricated by applying an exponential voltage decrease at the end of the anodization process. The time constant $\eta$ of the exponential voltage function has been used to control the average thickness and the thickness distribution of the barrier layer at the bottom of the pores of the alumina structure. Depending on the $\eta$ value, the thickness distribution of the barrier layer can be made very uniform or highly scattered, which allows us to subsequently fine tune the electrodeposition yield of nickel nanoparticles/nanowires at low voltage. As an illustration, the pore filling percentage with $\mathrm{Ni}$ has been varied, in a totallyreproducible manner, between $\sim 3$ and $100 \%$. Combined with the ability to vary the porediameter and repetition step over $\sim 2$ orders of magnitude (by varying the anodization voltageand electrolyte type), the control of the pore filling percentage with metal particles/nanowires could bring novel approaches for the organization of nanoobjects.
\end{abstract}

\section{Introduction}

The introduction of nanomaterials in device applications implies control over their spatial organization at the nanometric scale. One of the promising approaches to avoid cost-intensive nano-lithography steps which are commonly employed in ultimate device fabrication is to use ordered templates. Among these, porous anodic alumina (PAA) templates have especially attracted interest as they present a very simple method to control a nano-arrangement. In 1995, Masuda et al set a milestone in the fabrication of PAA templates since a perfectly hexagonal self-ordered pore structure was reported [1]. Following this work, research focused on the variation of the main properties of such ordered PAA, i.e., the cell size, pore diameter and pore depth, by changing the anodization voltage, the acidic solution, and the anodization time [2-4]. Due to the easy and versatile variation of their geometrical properties, PAA templates have been used for a variety of applications. In particular, PAA has served as a template for the growth of metal nanowires, silicon nanowires (SiNWs) and carbon nanotubes (CNTs) [5-7], whereas the use of PAA has also appeared useful for devices and characterization measurements $[8,9]$.

However, the pore density control, regardless of the pore diameter, is still an open issue. In contrast to the investigations performed so far $[3,4,10]$, we propose here to control the density of pores filled with metal (by electrodeposition) rather than the pore density itself. This could offer new possibilities of surface organization by means of PAA templates, since the controlled electrodeposition of various metals inside pores is the basic step for growing arrays of metal nanowires as well as for the growth of SiNWs or CNTs (by chemical vapour deposition), where metal seeds are used as catalysts. 
In our approach we focus on the treatment of the barrier layer which is naturally present at the bottom of the pores in PAA. This barrier layer, consisting of alumina, is highly resistive and thus may hinder the electrodeposition process inside the pores. The barrier layer has been widely investigated in the literature [11-13], and its thickness, $d_{\mathrm{bl}}$, can be estimated by the equation $d_{\mathrm{bl}}=k U$, where $k \sim 1.2 \mathrm{~nm} \mathrm{~V}^{-1}$ at room temperature and $U$ is the anodization voltage expressed in volts. One important feature is the slight variation of $d_{\mathrm{bl}}$ from one pore to the next, which is mainly due to local defects/impurities in the $\mathrm{Al}$ material. Thus, the resistance during electrodeposition varies depending on the pores, which influences the deposition behaviour, since electrodeposition preferably occurs in those pores exhibiting a thinner (i.e., a less resistive) barrier layer $[14,15]$. Here, we show that, with a precisely controlled voltage decrease at the end of the anodization process, the barrier layer is thinned down with either an amplification or a smoothening of the variation of the barrier layer thickness over the sample. This results in an effective control of the distribution of filled pores during the subsequent electrodeposition process.

\section{Experimental details}

The PAA membranes were fabricated by anodization, which was performed on $3 \mu \mathrm{m}$ thick $\mathrm{Al}$ films. The $\mathrm{Al}$ was sputtered on a $500 \mathrm{~nm}$ thick $\mathrm{SiO}_{2}$ layer grown on $\mathrm{Si}$ and subsequently annealed at $450{ }^{\circ} \mathrm{C}$ for $2 \mathrm{~h}$ under flowing $\mathrm{N}_{2}$. For anodization and electrodeposition, a special mechanical set-up was used so that for each sample a precisely defined area of $0.38 \mathrm{~cm}^{2}$ was exposed to the acidic solutions. During all electrochemical steps, the solutions were heavily agitated by a stir-bar which was positioned above the sample surface. The anodization was carried out in $0.3 \mathrm{M}$ oxalic acid by controlling the anodization voltage with a programmable power supply (Itech Electronic IT6834). The temperature of the electrochemical baths was precisely maintained at $25^{\circ} \mathrm{C}$, whether for anodization or electrodeposition. For the preparation of the PAA membranes, a one-step anodization process at $40 \mathrm{~V}$ was used for $500 \mathrm{~s}$, in order to create a porous structure exhibiting a pore diameter of about $40 \mathrm{~nm}$ and a thickness of about $1.5 \mu \mathrm{m}$. At the end of the constant voltage step, a precisely controlled voltage decrease was applied to the samples. The final value of the anodization voltage was fixed at $5.0 \mathrm{~V}$, which corresponds to a final barrier layer thickness of about $6 \mathrm{~nm}$. Then, the PAA samples were etched in $3 \mathrm{wt} \% \mathrm{H}_{3} \mathrm{PO}_{4}$ for $25 \mathrm{~min}$ at $30{ }^{\circ} \mathrm{C}$. Finally, Ni was electrodeposited with $5 \mathrm{~ms}$ pulses at various negative voltages, using the so-called Watts bath as electrolyte, (a mixture of $500 \mathrm{~g} \mathrm{l}^{-1} \mathrm{NiSO}_{4}, 45 \mathrm{~g} \mathrm{l}^{-1} \mathrm{NiCl}_{2}$ and $45 \mathrm{~g} \mathrm{l}^{-1} \mathrm{H}_{3} \mathrm{BO}_{3}$ ). The electrodeposition voltage was controlled in the potentiostatic mode using a HEKRA PG310 voltage supply. We used the HEKRA PG310 in the three-electrode mode with an $\mathrm{Ag} / \mathrm{AgCl}$ reference electrode and a graphite electrode with a surface of $12.6 \mathrm{~cm}^{2}$ as the counter electrode. As the driving programme, the POTPULSE programme created by HEKRA was used. We used a Hitachi S4800 FEG high resolution SEM for sample observation. To determine the pore diameter and filling density from the SEM images, we used the software 'Image J' (a public domain programme developed at the Research Services Branch (RSB) of the National Institutes of Health (NIH), USA).

\section{Results and discussion}

The approach we present here is based on the precise control of the barrier layer thickness, thus allowing us to change the density of the filled pores for a given low electrodeposition voltage. For this, the barrier layer is thinned by decreasing the voltage at the end of the anodization process. Such voltage decrease has already been reported [14, 18], but it has never been studied precisely and systematically. The voltage decrease at the end of the anodization process influences the porous structure in two ways: on the one hand, it thins down the barrier layer, and on the other hand, it induces a change in the pore density (the latter being a function of the anodization voltage [2]), leading to a tree-like branched structure [16-18]. This tree-like structure is due to the fact that at each step, during the voltage decrease, a new equilibrium state is established with a corresponding specific pore density (number of pores per surface unit), a specific anodization current and a specific barrier layer thickness. This can be clearly observed in figure 1(a): with each voltage drop, the current decreases instantly but then increases again until a new equilibrium state is reached.

In a first series of experiments, the stepwise voltage decrease was smoothened by applying a continuously decreasing function, in order to avoid discrete constant voltage drops. Since the barrier layer thickness and thus the resistance of the electrical circuit is proportional to the applied anodization voltage, the voltage decrease at any particular time should always be proportional to the previously applied voltage. This can be expressed by an exponential decrease function:

$$
\mathrm{d} U / \mathrm{d} t=\text { const } \times U \Rightarrow U=U_{0} \exp (-\eta t)+U^{\mathrm{t}} .
$$

The time constant $\eta$ gives the rate of the voltage decrease. The higher the $\eta$ value, the higher the decreasing rate. $U_{0}$ and $U^{t}$ are free parameters whose ideal values depend on the chosen constant voltage of the PAA fabrication procedure. In our study, the parameters were fixed at $50 \mathrm{~V}$ for $U_{0}$ and $-10 \mathrm{~V}$ for $U^{t}$. These values are the result of an optimization which will not be discussed here.

For all samples, a continuous exponential decrease of the anodization voltage was applied down to $5.0 \mathrm{~V}$. This final voltage was maintained for $20 \mathrm{~s}$. Two different regimes could be evidenced from our experiments.

(i) In the case of a very slow voltage decrease, the anodization process is always close to the equilibrium state. Branches are formed at the pore bottom (as already discussed) and the final anodization current is quite high (i.e., $430 \mu \mathrm{A} \mathrm{cm}^{-2}$, figure $1(\mathrm{~b})$ ), reflecting an overall low resistance value of the parallel-connected barrier layers at the bottom of the pores. This can be evidenced by a subsequent electrodeposition process (at low voltage, here $-4.2 \mathrm{~V}$ ) which yields a uniform, real $100 \%$ filling of the pores; see figure 2(a). For the sample shown in 

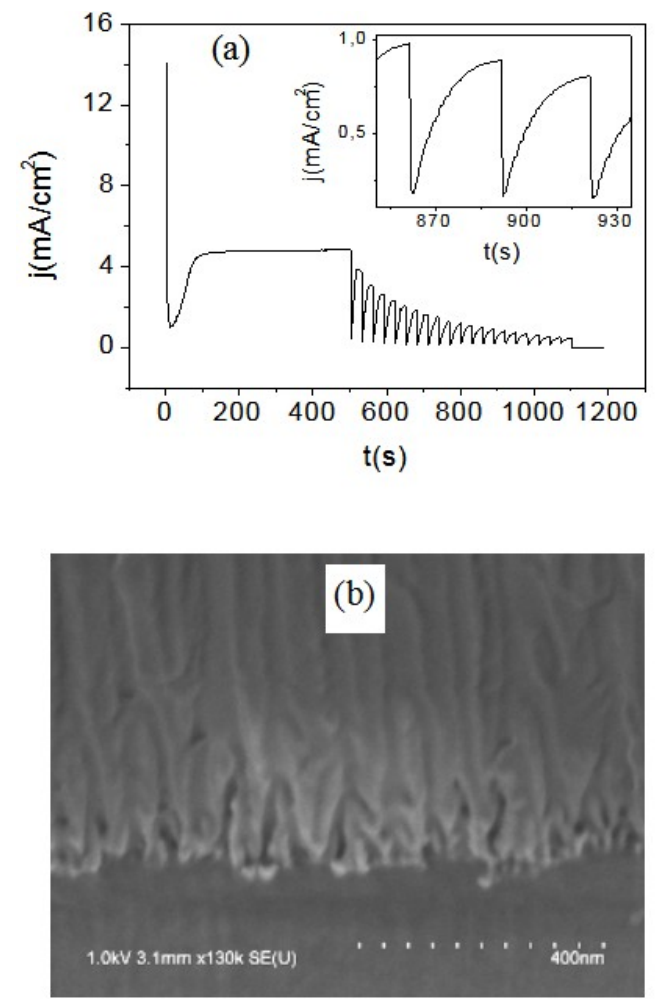
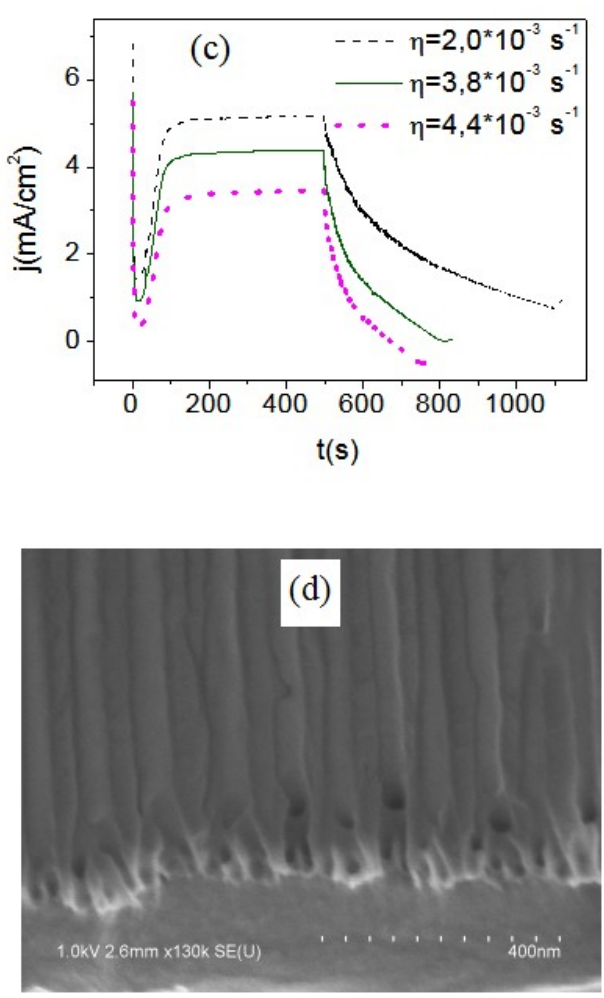

Figure 1. Effect of the voltage decrease at the end of the anodization process. (a) Current response as a function of time during anodization. Once anodization is completed, the voltage is decreased stepwise, each time by $10 \%$, and each voltage step is kept constant for 30 s. The inset shows a magnification of the current response between $\sim 850$ and $950 \mathrm{~s}$. (b) SEM image of a $45^{\circ}$ tilted sample processed according to the conditions presented in (a). Note the highly branched structure of the pores near the bottom. (c) Influence of the time constant of an exponential decrease function on the current behaviour; the three curves have been split for the sake of clarity, but in each case the plateau region corresponds to $4 \mathrm{~mA} \mathrm{~cm}-2$. (d) SEM picture of the pore bottom. The image shows the $45^{\circ}$ tilted sample processed with the fastest voltage decrease, i.e. $\eta=4.4 \times 10^{-3} \mathrm{~s}^{-1}$. Note that there is little branching in this case.

this figure, the alumina membrane was dissolved in a mixture of $1.7 \mathrm{wt} \% \mathrm{CrO}_{3}$ and $6 \mathrm{wt} \% \mathrm{H}_{3} \mathrm{PO}_{4}$ at $60{ }^{\circ} \mathrm{C}$ after electrodeposition, allowing the direct observation of the deposited Ni particles.

(ii) If the voltage decrease is faster, the anodization process is in permanent non-equilibrium. Consequently, the formation of branches at the pore bottom is strongly suppressed (figure 1(b)) and the final anodization current (at low voltage) becomes very low $\left(3.0 \mu \mathrm{A} \mathrm{cm}^{-2}\right)$, reflecting the fact that few barrier layers are now conducting. In this case, the barrier layer thicknesses differ significantly from the expected value of $6 \mathrm{~nm}$, which will be shown and discussed in the following.

Generally, the formation of the PAA structure schematically results from a field-dependent equilibrium between oxide formation at the oxide/metal interface and field-assisted oxide dissolution in the acid solution at the oxide/electrolyte interface [19]. Taking this into account, the two different behaviours that we have observed can be explained as follows: when the anodization voltage is decreased, the thickness of the barrier layer is at first larger than the equilibrium thickness, thus inhibiting current flow (see the current versus time behaviour of figure 1(a)) and the formation of new alumina. If a sufficient amount of time is allowed, because of the ongoing dissolution of alumina at the pore bottom (there is still an applied electric field there), a new equilibrium between anodization voltage and barrier layer thickness is reached and the anodization process can start again, like in a feedback loop. If the voltage is decreased faster, the equilibrium state is never attained, i.e. the barrier layer is always thicker than in the equilibrium state (the voltage decrease rate has to match the dissolution rate at the bottom of the pores). The anodization process will stop as soon as the resistance of the remaining barrier layer cannot be overcome by the voltage actually applied. This means that in those pores whose initial barrier layer is thicker, the anodization process already stops, while it is still continuing in those with a thinner initial barrier layer. Consequently, the variation of the barrier layer thickness at the end of the voltage decrease is much higher than the one in the case of the equilibrium state. In other words, a proper choice of $\eta$ will result in an amplification of the slight thickness heterogeneity of the barrier layer after the constant voltage oxidation step. This is evidenced by the result of low voltage $(-4.2 \mathrm{~V})$ electrodeposition: the filling percentage is drastically decreased for higher $\eta$ values (figures 2(b), (c)). It is clear that the influence of the time constant becomes highly sensitive when the voltage decrease rate comes close to a limit value where there is not enough time for any barrier thinning by dissolution. This can also be observed in figures 2(b) and (c), where the filling density changes from $5.5 \times 10^{10}$ to $2.3 \times 10^{9} \mathrm{~cm}^{-2}$ when $\eta$ is changed from $3.8 \times 10^{-3}$ to $4.4 \times 10^{-3} \mathrm{~s}^{-1}$. 
(a)
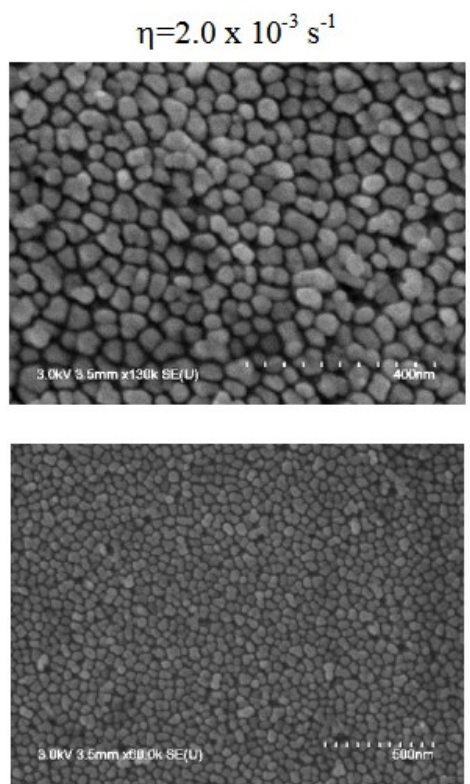

density of filled pores: (b)
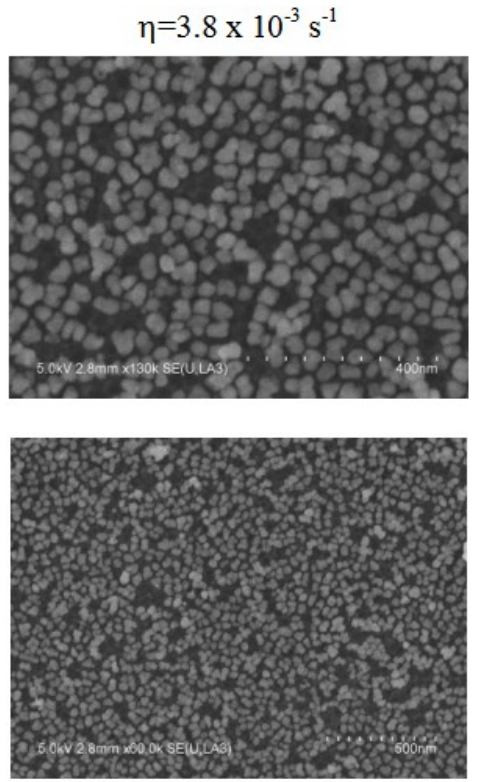

density of filled pores: (c)
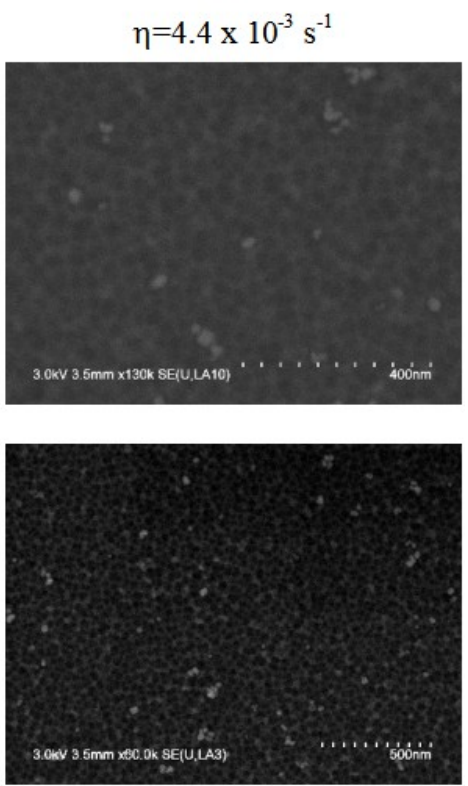

density of filled pores:

$2.310^{9} \mathrm{~cm}^{-2}$$$
2.310^{9} \mathrm{~cm}^{-2}
$$

$$
7.1 \times 10^{10} \mathrm{~cm}^{-2}
$$$$
5.5 \times 10^{10} \mathrm{~cm}^{-2}
$$

Figure 2. Influence of the time constant $\eta$ on the distribution of the deposited Ni particles inside the pores (electrodeposition voltage $=-4.2 \mathrm{~V}$ ). The SEM images show top views of the samples after selective etching of the alumina membrane.

To further evidence the key role of the time constant value for controlling the thickness distribution of the barrier layer and thus the nanopore filling ratio, we investigated the current response $I_{\text {ed }}$ of various membranes (i.e., membranes synthesized with different time constants) as a function of the applied electrodeposition voltage $U_{\text {ed }}$. For this purpose, $U_{\text {ed }}$ was varied from 2.5 to $6.5 \mathrm{~V}$ by applying voltage pulses of $5 \mathrm{~ms}$ followed by a delay time of $90 \mathrm{~ms}$. A typical current response of the applied voltage pulse is shown in figure 3(a). Taking into account only the maximum current values reached during the $5 \mathrm{~ms}$ voltage pulses, we could plot the current response curve for the applied electrodeposition voltage in the range from 2.5 to $6.5 \mathrm{~V}$. Such a current response curve is shown in figure 3(b).

The $I-V$ graph can be divided into three different regimes. In the first regime, the voltage is not high enough to overcome the barrier layer and to reduce the $\mathrm{Ni}$ ions at the same time.

Only a very low leakage current is observed, which slightly increases linearly (about $0.4 \mathrm{~mA} \mathrm{~V}^{-1}$ ) with the applied voltage.

In the second regime, the applied voltage is just overcoming the threshold value, which is determined by the barrier layer resistance, the reduction potential of $\mathrm{Ni}$ and the electrolyte resistance. In the third regime, the linear current increase corresponds to the reduction and deposition of $\mathrm{Ni}$ at the pore bottom, which depend linearly on the applied voltage due to the combined resistance of the barrier layer and of the electrolyte.

Of course, the measured current response is the sum of all currents flowing at the pore bottom of the parallel-connected pores of the alumina membrane. In this sense, especially the second and the third regime deliver information about the thickness distribution of the barrier layer. If the barrier layer thickness is homogeneous over the sample, the threshold voltage is identical inside all pores. Thus, the change from the first linear regime (leakage current) to the second one (reduction of Ni ions) is abrupt, and the current curve shows a sharp bend. If the barrier layer thickness varies over the sample, the threshold voltage is not the same over the whole sample area. Thus, the change between the two different linear regimes takes place over a wider voltage range as the number of pores in, which $\mathrm{Ni}$ ions are reduced and $\mathrm{Ni}$ is deposited, increases with the increasing electrodeposition voltage.

In our experiments, we have measured the current response of the electrodeposition voltage function for two different samples: one after a slow $\left(\eta=2.0 \times 10^{-3} \mathrm{~s}^{-1}\right)$ voltage decrease, and the other after a fast $(\eta=4.4 \times$ $10^{-3} \mathrm{~s}^{-1}$ ) voltage decrease (figure 3(c)). The low $\eta$ value

yields a sharp transition between the linear regimes, which takes place between $\sim-3.3$ and $-3.8 \mathrm{~V}$. The high $\eta$ value yields a much larger transition region, in the range of -3.6 to $-4.9 \mathrm{~V}$. This clearly confirms the strong influence of the time constant of the exponential decrease on the distribution of the barrier layer thickness. Besides, in the case of the high $\eta$ value, the transition regime (regime 2) is strongly extended to higher voltage values (in comparison to the low $\eta$ case), which strengthens the explanations given above for the influence of the time constant on the barrier layer thickness distribution: as, during a fast voltage decrease, only those pores with a very thin initial barrier layer thickness can follow the anodization process until the end, the majority of the pores exhibit a barrier layer which is thicker than the expected value of $6 \mathrm{~nm}$. This leads to an extension of the transition region in the $I-V$ 
(a)

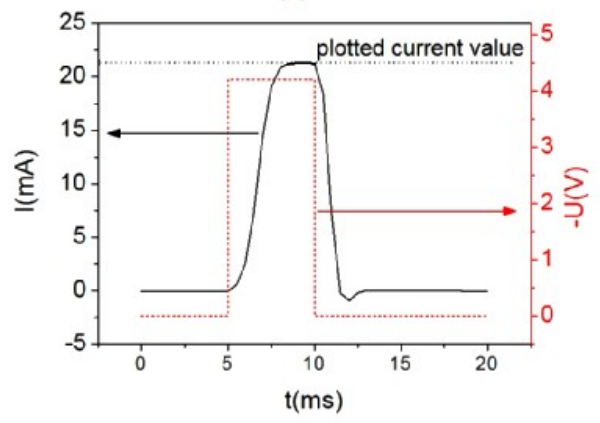

(b)

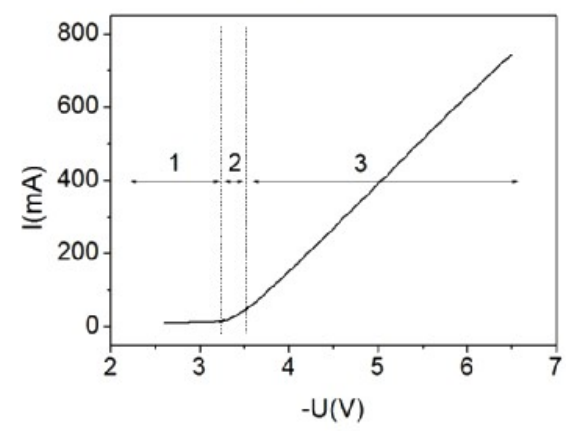

(c)
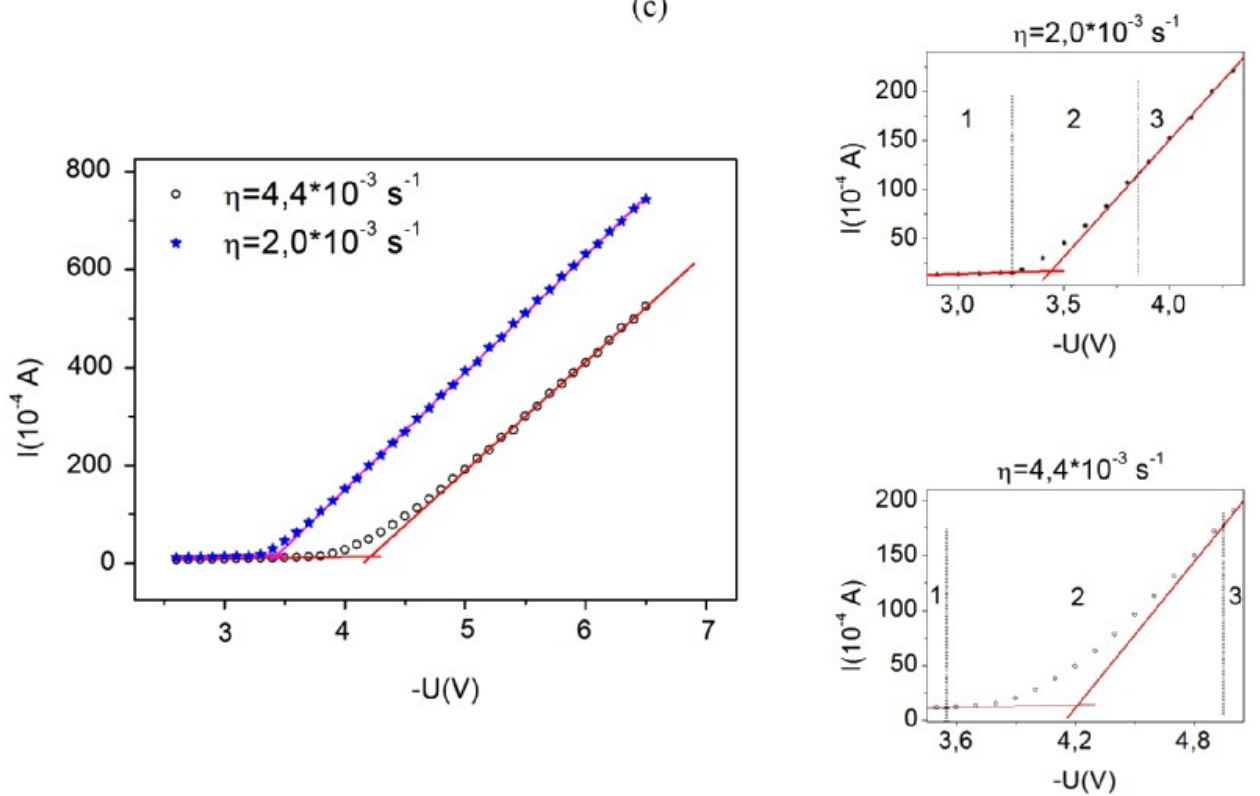

Figure 3. Electrodeposition inside alumina membranes. (a) A typical voltage pulse and the corresponding current response. For the $I-V$ plot, only the maximum values of both the voltage and the current are taken into account. (b) Typical current-voltage plot of a membrane during electrodeposition. The three different regimes are discussed in the text. (c) Current-voltage plots for two membranes synthesized with $\eta=2.0 \times 10^{-3} \mathrm{~s}^{-1}$ and $\eta=4.4 \times 10^{-3} \mathrm{~s}^{-1}$. The enlargements focus on the transition region between the first and third regimes. The first and third regimes are fitted with a linear function in order to better visualize the transition between these two regimes.

curve towards higher electrodeposition voltages due to higher threshold voltages inside the major part of the pores.

The previously given explanation implies that the average barrier layer thickness and thus the average electrical resistance during the electrodeposition process is higher for samples with a faster exponential voltage decrease. This can be seen in figure 3(c) in the third regime of the $I-V$ graph, where the slope of the linear increase during the electrodeposition is $22.1 \mathrm{~mA} \mathrm{~V}^{-1}$ for $\eta=4.4 \times 10^{-3} \mathrm{~s}^{-1}$ and $23.8 \mathrm{~mA} \mathrm{~V}-1$ for $\eta=$ $2.0 \times 10^{-3} \mathrm{~s}^{-1}$, which corresponds to a resistance of $45.2 \mathrm{Q}$ and $42.0 \mathrm{Q}$, respectively. Taking into account the geometry of our set-up and a resistivity of the electrolyte (Watts bath) of about $10 Q \mathrm{~cm}$, we calculated a series resistance of about $14 Q$ inside the electrolyte. This resistance has to be subtracted from the measured values in order to obtain the effective resistance of the barrier layer, which we thereby determined to be $31.2 Q$ $\left(\eta=4.4 \times 10^{-3} \mathrm{~s}^{-1}\right)$ and $28.0 \mathrm{Q}\left(\eta=2.0 \times 10^{-3} \mathrm{~s}^{-1}\right)$. This means a shift of the average resistance of the barrier layer between the two different samples of $11 \%$.
An interesting effect can be observed when the applied electrodeposition voltage is increased to a value high enough to overcome the barrier layer resistance in all pores (namely $-5.5 \mathrm{~V}$ instead of the $-4.2 \mathrm{~V}$ during the previous experiments). Figure 4 summarizes the results obtained for two of the time constant values reported above: $\eta=2.0 \times 10^{-3} \mathrm{~s}^{-1}$ and $\eta=4.4 \times 10^{-3} \mathrm{~s}^{-1}$. For the low $\eta$ value, a high density, homogeneous deposition of $\mathrm{Ni}$ nanowires (NiNWs) with uniform length is observed (figure 4(a)), whilst for the higher $\eta$ value, the deposited NiNWs show a significant length variation (figure 4(b)). We relate the length variation of the NiNWs to the barrier layer thickness variation, since the deposition rate depends on the thickness of the barrier layer: the lower the barrier layer thickness, the lower the resistance and thus the higher the electrodeposition current. The deposition rate depends directly on the deposition current, and as a consequence, from the length variation of the NiNWs, the barrier layer distribution can be deduced, which confirms the results given above. 
(a)

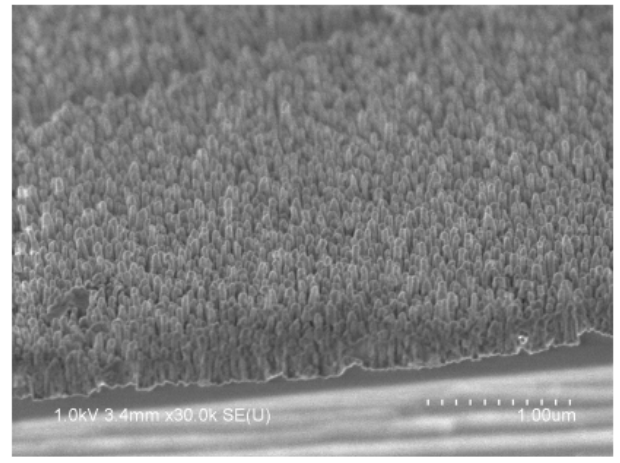

$\eta=2.0 \times 10^{-3} \mathrm{~s}^{-1}$ (b)

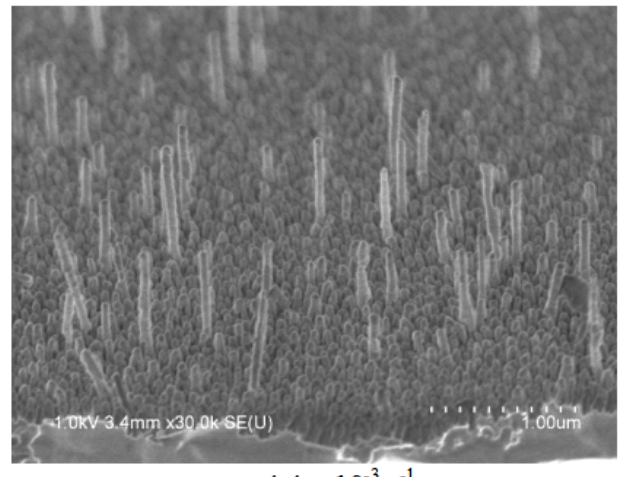

$\eta=4.4 \times 10^{-3} \mathrm{~s}^{-1}$

Figure 4. Influence of the time constant $\eta$ on the uniformity of the electrodeposition process inside PAA membranes for an electrodeposition voltage of $-5.5 \mathrm{~V}$. SEM images after the selective etching of the membrane (the samples are tilted $45^{\circ}$ ). Note the large height variations of the Ni nanowires corresponding to the higher $\eta$ value.

\section{Conclusion}

In conclusion, the filling density inside a PAA template can be varied by a precise control of the function (time constant) applied for the voltage decrease at the end of the anodization process. The density of the deposited Ni nanowires has thus been varied between $7.1 \times 10^{10} \mathrm{~cm}^{-2}$ and $2.3 \times 10^{9} \mathrm{~cm}^{-2}$, which corresponds to a filling rate of $100 \%$ to $3 \%$ respectively. This offers a new possibility for the organization of nanoobjects using PAA templates, which can be useful for different kinds of applications, in particular for the use of PAA as a support for field emission devices.

\section{Acknowledgments}

This work was supported by the EU project CANDICE, the ANR project NANOBIOSENSOR and the Samsung Electronics 'NanodiX' Chair. The authors would like to thank P Allongue, J-N Chazalviel and F Ozanam for helpful discussions concerning the interpretation of the $I-V$ curves.

\section{References}

[1] Masuda H and Fukuda K 1995 Science 2681466

[2] Li A P, Müller F, Birner A, Nielsch K and Gösele U 1998 J. Appl. Phys. 846023
[3] Lee W, Ji R, Gösele U and Nielsch K 2006 Nat. Mater. 5741

[4] Zhang F, Liu X, Pan C and Zhu J 2007 Nanotechnology 18345302

[5] Rabin O, Herz P R, Lin Y M, Akinwande A I, Cronin S B and Dresselhaus M S 2003 Adv. Funct. Mater. 13631

[6] Lew K K, Reuther C, Carim A H and Redwing J M 2002 J. Vac. Sci. Technol. B 20389

[7] Maschmann M R, Franklin A D, Amama P B, Zakharov D N, Stach E A, Sands T D and Fisher T S 2006 Nanotechnology 173925

[8] Yang C J, Chen C C and Shieh J M 2006 J. Appl. Phys. 100104302

[9] Dayen J F, Rumyantseva A, Ciornei C, Wade T L, Wegrowe J E, Pribat D and Cojocaru C S 2007 Appl. Phys. Lett. 90173110

[10] Chu S Z, Wada K, Inoue S, Isogai M and Yasumori A 2005 Adv. Mater. 172115

[11] Tian M, Xu S, Wang J, Kumar N, Wertz E, Li Q, Campbell P M, Chan M H and Mallouk T E 2005 Nano Lett. 5697

[12] Brevnov D A, Rao G V, Lopez G P and Atanassov P B 2004 Electrochim. Acta 492487

[13] Saedi A and Ghorbani M 2005 Mater. Chem. Phys. 91417

[14] Nielsch K, Müller F, Li A P and Gösele U 2000 Adv. Mater. 12582

[15] Gerein N J and Haber J A 2005 J. Phys. Chem. B 10917372

[16] Li J, Papadopoulos C and Xu J 1999 Nature 402253

[17] Meng G, Jung Y J, Cao A, Vajtai R and Ajayan P M 2005 Proc. Natl Acad. Sci. 1027074

[18] Cheng W, Steinhart M, Gösele U and Wehrspohn R B 2007 J. Mater. Chem. 173493

[19] Jessensky O, Müller F and Gösele U 1998 Appl. Phys. Lett. 721173 